\title{
Simulating non-prenex cuts in quantified propositional calculus
}

\author{
Emil Jeřábek* Phuong Nguyen ${ }^{\dagger}$
}

May 2, 2011

\begin{abstract}
We show that the quantified propositional proof systems $G_{i}$ are polynomially equivalent to their restricted versions that require all cut formulas to be prenex $\Sigma_{i}^{q}$ (or prenex $\left.\Pi_{i}^{q}\right)$. Previously this was known only for the treelike systems $G_{i}^{*}$.
\end{abstract}

\section{Introduction}

The quantified propositional sequent calculus $G$ and its subsystems $G_{i}$ and $G_{i}^{*}$ were introduced in $[4,5,3]$. The systems $G_{i}$ and $G_{i}^{*}$ were slightly redefined in [1] so that they can prove all quantified propositional tautologies. Under the new definition some subtle but interesting issues arise when we look at $G_{i}$-proofs of tautologies that are not in $\Sigma_{i}^{q} \cup \Pi_{i}^{q}$. For example, can the target formulas be restricted to be the constants $\perp, \top$ without increasing the size of the proofs by more than a polynomial factor? Another question is whether the cut formulas can be restricted to be prenex formulas. More precisely, $G_{i}$ is defined so that it allows cuts on $\Sigma_{i}^{q} \cup \Pi_{i}^{q}$-formulas, where these classes have a fairly permissive definition (e.g., they are closed under $\wedge$ and $\vee$ ). Morioka [6] (cf. [2, Thm. VII.4.7]) has shown that in the case of the treelike fragments $G_{i}^{*}$, we can restrict cut formulas to prenex $\Sigma_{i}^{q}$, up to polynomial simulation. The purpose of this note is to generalize this result to daglike $G_{i}$ and thus answer the second question above, see Theorem 2.3 below.

Morioka proves his result essentially by transforming the given $G_{i}^{*}$-proof so that all cut formulas and their ancestors become prenex. More precisely, quantifiers inside each cut formula are successively moved outward by standard prenexation rules, and these changes are propagated to its ancestors up to the point where the quantifier is introduced. For this transformation the assumption that the proof is treelike is crucial. In particular, it is not directly applicable to the case of daglike proofs, because here a sequent may be used in

\footnotetext{
*Institute of Mathematics of the Academy of Sciences, Žitná 25, 11567 Praha 1, Czech Republic, email: jerabek@math.cas.cz, URL: http://math.cas.cz/ jerabek. Supported by Institutional Research Plan AV0Z10190503, grant IAA100190902 of GA AV ČR, project 1M0545 of MŠMT ČR, and a grant from the John Templeton Foundation.

${ }^{\dagger}$ School of Computer Science, McGill University, 3480 University Street, Montreal, Quebec, Canada H3A 2A7, email: pnguyen@cs.toronto.edu, URL: http://www.cs.toronto.edu/ pnguyen. Supported by an NSERC Postdoctoral Fellowship.
} 
several different inferences, and so modifying a formula of a sequent $\mathcal{S}$ that is used as a cut formula may invalidate other inferences that involve $\mathcal{S}$.

In order to make it work for daglike proofs, we perform instead a uniform global transformation of the whole proof: we modify every sequent in $\pi$ by replacing each $\Sigma_{i}^{q} \cup \Pi_{i}^{q}$ formula $\psi$ by a prenex $\Sigma_{i}^{q}$ formula $\psi^{\prime}$, which is a prenex form of either $\psi$ or $\neg \psi$ (depending on $\psi$ and where it appears - antecedent or succedent.) Note that we do not modify $\Sigma_{i}^{q} \cup \Pi_{i}^{q}$ subformulas of other formulas in $\pi$. The main task now is to show that the transformed sequents can be augmented to a valid proof of $\varphi$, which boils down essentially to providing short $G_{i}^{*}$-proofs of the equivalence of a $\Sigma_{i}^{q}$ (or $\Pi_{i}^{q}$ ) formula to its prenex forms. This turns out to be rather technically involved, and it is the main contribution of this paper.

\section{Definitions}

We refer to $[3,2]$ for basic definitions of quantified propositional formulas (or just formulas) and the sequent calculus $G$. In short, a $G$-proof of a sequent $\mathcal{S}$ is a sequence of sequents

$$
\mathcal{S}_{1}, \mathcal{S}_{2}, \ldots, \mathcal{S}_{m}
$$

where $\mathcal{S}_{m}=\mathcal{S}$, and each $\mathcal{S}_{k}$ follows from some of the sequents $\mathcal{S}_{k^{\prime}}, k^{\prime}<k$, by an inference rule. The proof is said to be treelike if every $\mathcal{S}_{l}$ is used exactly once to derive another $\mathcal{S}_{k}$ (where $k>l$ ). (Generally proofs are daglike.) The rules of $G$ are listed below:

initial rule:

$$
i \underset{\varphi \longrightarrow \varphi}{\longrightarrow}
$$

weakening rules:

$$
w-\mathrm{l} \frac{\Gamma \longrightarrow \Delta}{\varphi, \Gamma \longrightarrow \Delta} \quad w-\mathrm{r} \frac{\Gamma \longrightarrow \Delta}{\Gamma \longrightarrow \Delta, \varphi}
$$

exchange rules:

$$
x-\mathrm{l} \frac{\Gamma_{1}, \varphi, \psi, \Gamma_{2} \longrightarrow \Delta}{\Gamma_{1}, \psi, \varphi, \Gamma_{2} \longrightarrow \Delta} \quad x-\mathrm{r} \frac{\Gamma \longrightarrow \Delta_{1}, \varphi, \psi, \Delta_{2}}{\Gamma \longrightarrow \Delta_{1}, \psi, \varphi, \Delta_{2}}
$$

contraction rules:

$$
c-\mathrm{l} \frac{\Gamma, \varphi, \varphi \longrightarrow \Delta}{\Gamma, \varphi \longrightarrow \Delta} \quad c-\mathrm{r} \frac{\Gamma \longrightarrow \Delta, \varphi, \varphi}{\Gamma \longrightarrow \Delta, \varphi}
$$

constant introduction rules:

$$
\perp-\mathrm{l} \underset{\perp \longrightarrow}{\longrightarrow} \quad \mathrm{T}-\mathrm{r} \underset{\longrightarrow}{\longrightarrow}
$$


$\neg$-introduction rules:

$$
\neg-1 \frac{\Gamma \longrightarrow \Delta, \varphi}{\neg \varphi, \Gamma \longrightarrow \Delta} \quad \neg-\mathrm{r} \frac{\varphi, \Gamma \longrightarrow \Delta}{\Gamma \longrightarrow \Delta, \neg \varphi}
$$

$\wedge$-introduction rules:

$$
\wedge-1 \frac{\varphi, \psi, \Gamma \longrightarrow \Delta}{(\varphi \wedge \psi), \Gamma \longrightarrow \Delta} \quad \wedge-\mathrm{r} \frac{\Gamma \longrightarrow \Delta, \varphi \quad \Gamma \longrightarrow \Delta, \psi}{\Gamma \longrightarrow \Delta,(\varphi \wedge \psi)}
$$

V-introduction rules:

$$
\vee \text {-l } \frac{\varphi, \Gamma \longrightarrow \Delta \quad \psi, \Gamma \longrightarrow \Delta}{(\varphi \vee \psi), \Gamma \longrightarrow \Delta} \quad \vee-\mathrm{r} \frac{\Gamma \longrightarrow \Delta, \varphi, \psi}{\Gamma \longrightarrow \Delta,(\varphi \vee \psi)}
$$

$\forall$-introduction rules:

$$
\forall-1 \frac{\varphi(x / \psi), \Gamma \longrightarrow \Delta}{\forall x \varphi, \Gamma \longrightarrow \Delta} \quad \forall-\mathrm{r} \frac{\Gamma \longrightarrow \Delta, \varphi(x / p)}{\Gamma \longrightarrow \Delta, \forall x \varphi}
$$

$\exists$-introduction rules:

$$
\exists-1 \frac{\varphi(x / p), \Gamma \longrightarrow \Delta}{\exists x \varphi, \Gamma \longrightarrow \Delta} \quad \exists-\mathrm{r} \frac{\Gamma \longrightarrow \Delta, \varphi(x / \psi)}{\Gamma \longrightarrow \Delta, \exists x \varphi}
$$

cut rule:

$$
\operatorname{cut} \frac{\Gamma \longrightarrow \Delta, \varphi \quad \varphi, \Gamma \longrightarrow \Delta}{\Gamma \longrightarrow \Delta}
$$

For the rules $\forall$-l and $\exists$-r, $\varphi(x / \psi)$ is the result of substituting $\psi$ for all free occurrences of $x$ in $\varphi$. The formula $\psi$ is called the target formula and may be any quantifier-free formula (i.e., with no bound variables; also called open below) that is free for substitution for $x$ in $\varphi$ (i.e., no free occurrence of $x$ in $\varphi$ is within the scope of a quantifier $Q y$ such that $y$ occurs in $\psi$ ). The variable $p$ in the rules $\forall$-r and $\exists-1$ must not occur free in the bottom sequent and is called an eigenvariable.

The size of a formula $\varphi,|\varphi|$, is the total number of symbols appearing in it. The size of a proof is the total size of all formulas in the proof. We say that a (quantified) propositional proof system $P_{1}$ p-simulates another system $P_{2}$, in notation $P_{2} \leq_{p} P_{1}$, if there is a polytime function $F$ so that for every $P_{2}$-proof $\pi$ of a sequent $\mathcal{S}, F(\pi)$ is a $P_{1}$-proof of $\mathcal{S}$. We say that $P_{1}$ and $P_{2}$ are p-equivalent, denoted by $P_{1} \equiv_{p} P_{2}$, if both $P_{1} \leq_{p} P_{2}$ and $P_{2} \leq_{p} P_{1}$.

The subsystems of $G$ are defined by restricting the set of cut formulas.

Definition 2.1 $\Sigma_{0}^{q}=\Pi_{0}^{q}=\hat{\Sigma}_{0}^{q}=\hat{\Pi}_{0}^{q}$ is the set of quantifier-free propositional formulas. For $i>0, \Sigma_{i}^{q}, \Pi_{i}^{q}, \hat{\Sigma}_{i}^{q}$, and $\hat{\Pi}_{i}^{q}$ are defined inductively as the smallest sets of quantified propositional formulas such that for all formulas $\varphi, \psi$ and variables $x$ :

(i) $\Sigma_{i-1}^{q} \cup \Pi_{i-1}^{q} \subseteq \Sigma_{i}^{q} \cap \Pi_{i}^{q}$, 
(ii) if $\varphi, \psi \in \Sigma_{i}^{q}$, then $\varphi \wedge \psi, \varphi \vee \psi, \exists x \varphi \in \Sigma_{i}^{q}$ and $\neg \varphi \in \Pi_{i}^{q}$,

(iii) if $\varphi, \psi \in \Pi_{i}^{q}$, then $\varphi \wedge \psi, \varphi \vee \psi, \forall x \varphi \in \Pi_{i}^{q}$ and $\neg \varphi \in \Sigma_{i}^{q}$,

(iv) $\hat{\Sigma}_{i-1}^{q} \cup \hat{\Pi}_{i-1}^{q} \subseteq \hat{\Sigma}_{i}^{q} \cap \hat{\Pi}_{i}^{q}$,

(v) if $\varphi \in \hat{\Sigma}_{i}^{q}$ then $\exists x \varphi \in \hat{\Sigma}_{i}^{q}$,

(vi) if $\varphi \in \hat{\Pi}_{i}^{q}$ then $\forall x \varphi \in \hat{\Pi}_{i}^{q}$.

That is, $\hat{\Sigma}_{i}^{q}$ and $\hat{\Pi}_{i}^{q}$ consist of prenex formulas in $\Sigma_{i}^{q}$ and $\Pi_{i}^{q}$, respectively.

Definition 2.2 Let $i \geq 0 . G_{i}, \hat{G}_{i}$, and $\hat{G}_{i}^{\prime}$ denote the fragments of the quantified propositional sequent calculus that only allow cuts on $\Sigma_{i}^{q} \cup \Pi_{i}^{q}$-formulas, $\hat{\Sigma}_{i}^{q}$-formulas, and $\hat{\Pi}_{i}^{q}$-formulas, respectively. For any fragment $P$ of the sequent calculus, $P^{*}$ denotes its treelike version.

The main result of this paper is:

Theorem 2.3 For any $i, G_{i} \equiv_{p} \hat{G}_{i} \equiv_{p} \hat{G}_{i}^{\prime}$ and $G_{i}^{*} \equiv_{p} \hat{G}_{i}^{*} \equiv_{p} \hat{G}_{i}^{\prime *}$.

As mentioned in the introduction, the statement about treelike proof systems is Morioka's theorem. However our proof here applies to both treelike and daglike systems.

\section{The proof}

First we fix our procedure for putting a formula in prenex form. We use the following auxiliary operations. Given a formula, the result of our prenexation procedure will be defined in Definition 3.4.

Definition 3.1 Fix an infinite set $X$ of variables. For any prenex formulas $\varphi, \psi$, we define prenex formulas $\varphi \bigotimes^{\exists} \psi, \varphi \bigotimes^{\forall} \psi, \varphi \bigotimes^{\exists} \psi, \varphi \bigotimes^{\forall} \psi$, and $\curvearrowright \varphi$ as follows:

(i) $\curvearrowright \varphi=\neg \varphi$ and $\varphi \bigcirc^{Q} \psi=\varphi \circ \psi$ if $\varphi, \psi$ are open,

(ii) $\curvearrowright Q x \varphi=\bar{Q} x \curvearrowright \varphi$,

(iii) $(Q x \varphi) \bigcirc^{Q} \psi=Q y\left(\varphi(x / y) \bigcirc{ }^{Q} \psi\right)$,

(iv) $\psi \bigcirc^{Q}(Q x \varphi)=Q y\left(\psi \bigcirc^{Q} \varphi(x / y)\right)$ if $\psi$ does not start with $Q$,

(v) $\varphi \bigcirc^{Q} \psi=\varphi \bigcirc^{\bar{Q}} \psi$ if neither $\varphi$ nor $\psi$ start with $Q$,

where $\circ \in\{\wedge, \vee\}, Q \in\{\exists, \forall\}, \bar{Q}$ denotes the dual quantifier to $Q$, and $y$ is the first variable from $X$ with no free occurrences in $\psi$ such that either $x=y$ or $y$ does not appear (free or bound) in $\varphi$. 
The $\emptyset^{Q}, \emptyset^{Q}$, and $\odot$ operators perform one prenexing step, namely they provide a prenex form of $\varphi \wedge \psi, \varphi \vee \psi$, or $\neg \varphi$ (resp.) if $\varphi$ and $\psi$ are already prenex. The superscript $Q$ indicates what kind of quantifiers we prefer to pull to the prefix first: for example, if $\varphi$ and $\psi$ are quantifier-free, then (up to renaming of variables) $(\exists x \varphi(x)) \mathbb{A}^{\exists}(\forall y \psi(y))=$ $\exists x \forall y(\varphi(x) \wedge \psi(y))$, and $(\exists x \varphi(x)) \mathbb{Q}^{\forall}(\forall y \psi(y))=\forall y \exists x(\varphi(x) \wedge \psi(y))$. This is needed to ensure that we do not introduce too many quantifier alternations in the prenex formula.

Lemma 3.2 Let $\varphi, \psi$ be prenex formulas, $Q \in\{\exists, \forall\}$, and $\circ \in\{\wedge, \vee\}$.

(i) $|\bigcirc \varphi|=1+|\varphi|,\left|\varphi \bigcirc^{Q} \psi\right|=1+|\varphi|+|\psi|$.

(ii) $\bigcirc \varphi$ and $\varphi \bigcirc^{Q} \psi$ are polynomial-time computable given $\varphi, \psi$.

(iii) If $\varphi, \psi \in \hat{\Sigma}_{i}^{q}$, then $\varphi \bigcirc^{\exists} \psi \in \hat{\Sigma}_{i}^{q}$ and $\bigcirc \varphi \in \hat{\Pi}_{i}^{q}$.

(iv) If $\varphi, \psi \in \hat{\Pi}_{i}^{q}$, then $\varphi \bigcirc^{\forall} \psi \in \hat{\Pi}_{i}^{q}$ and $\curvearrowright \varphi \in \hat{\Sigma}_{i}^{q}$.

(v) $\varphi \bigcirc^{Q} \psi$ is equivalent to $\varphi \circ \psi$, and $\odot \varphi$ is equivalent to $\neg \varphi$.

Proof: By induction on the length of $\varphi, \psi$.

Lemma 3.3 Given prenex formulas $\varphi, \psi$ and $Q \in\{\exists, \forall\}$, there are polynomial-time constructible treelike cut-free proofs of the sequents

$$
\begin{aligned}
& \varphi, \psi \longrightarrow \varphi \mathbb{D}^{Q} \psi \\
& \varphi\left(\mathbb{A}^{Q} \psi \longrightarrow \varphi\right. \\
& \curvearrowright\left(\varphi \triangle^{Q} \psi\right) \longrightarrow(\varphi, \curvearrowright \psi \\
& \varphi\left(\mathbb{(}^{Q} \psi \longrightarrow \psi\right. \\
& \text { (ㄱ) } \bigcirc\left(\varphi\left(\mathbb{1}^{Q} \psi\right)\right. \\
& \varphi \nabla^{Q} \psi \longrightarrow \varphi, \psi \\
& \ominus \psi \longrightarrow \ominus\left(\varphi \otimes^{Q} \psi\right) \\
& \varphi \longrightarrow \varphi \nabla^{Q} \psi \\
& \bigcirc \varphi, \curvearrowright \psi \longrightarrow \curvearrowright\left(\varphi \nabla^{Q} \psi\right) \\
& \psi \longrightarrow \varphi \nabla^{Q} \psi \\
& \text { (ᄀ) }\left(\varphi \nabla^{Q} \psi\right) \longrightarrow \ominus \varphi \\
& \varphi, \curvearrowright \varphi \longrightarrow \\
& \ominus\left(\varphi \nabla^{Q} \psi\right) \longrightarrow \ominus \psi \\
& \varphi \longrightarrow \curvearrowright \bigcirc \varphi \\
& \curvearrowright \bigcirc \varphi \longrightarrow \varphi
\end{aligned}
$$

Proof: By induction on the complexity of $\varphi, \psi$. For example, we will treat the sequents

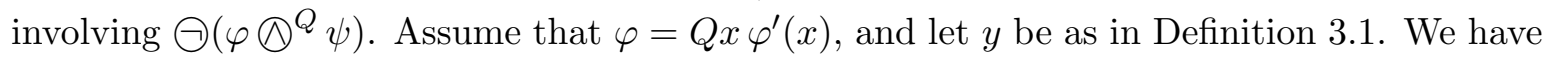
$\curvearrowright \varphi=\bar{Q} x \ni \varphi^{\prime}(x)$ and $\curvearrowright\left(\varphi \otimes^{Q} \psi\right)=\bar{Q} y \bigcirc\left(\varphi^{\prime}(y) \bigotimes^{Q} \psi\right)$. By the induction hypothesis, we can construct proofs of the sequents

$$
\begin{gathered}
\ominus\left(\varphi^{\prime}(y) \rrbracket^{Q} \psi\right) \longrightarrow \ominus \varphi^{\prime}(y), \ominus \psi, \\
\ominus \varphi^{\prime}(y) \longrightarrow \ominus\left(\varphi^{\prime}(y) \rrbracket^{Q} \psi\right), \\
\ominus \psi \longrightarrow \ominus\left(\varphi^{\prime}(y) \rrbracket^{Q} \psi\right) .
\end{gathered}
$$

We derive $\curvearrowright\left(\varphi\left(\mathbb{Q}^{Q} \psi\right) \longrightarrow(\neg \varphi, \bigcirc \psi\right.$ using one of the proofs

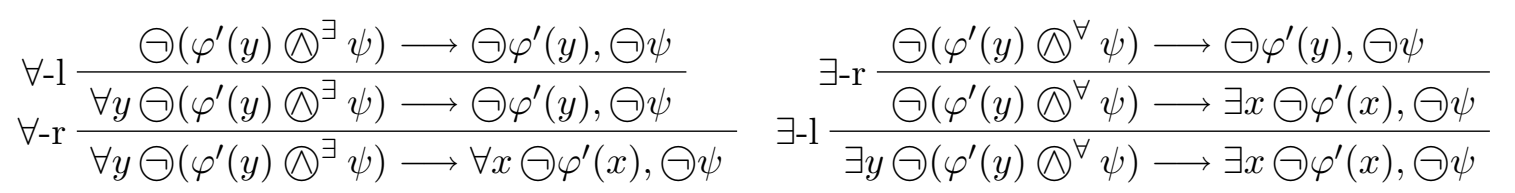


depending on $Q$. Similarly, we derive

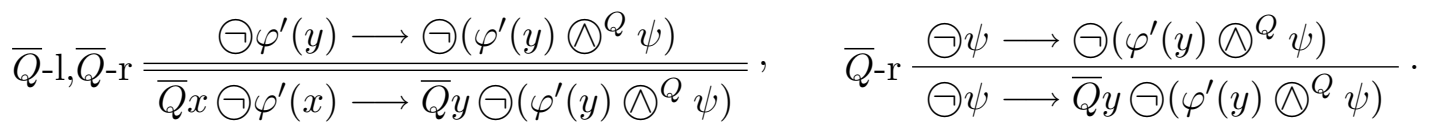

The other cases are similar.

We can now define the prenexation of any formula. As above, we will define two prenex forms to each formula, depending on the preference for what kind of quantifiers to pull out first. This is necessary, as for some formulas there does not exist a single prenex form preserving the complexity classification of the formula. For example, the formula $\chi=\exists x \varphi(x) \wedge \forall y \psi(y)$, where $\varphi$ and $\psi$ are open, is $\Sigma_{2}^{q} \cap \Pi_{2}^{q}$, and it is equivalent to a $\hat{\Sigma}_{2}^{q}$-formula $\chi^{\exists}=\exists x \forall y(\varphi(x) \wedge$ $\psi(y))$ and to a $\hat{\Pi}_{2}^{q}$-formula $\chi^{\forall}=\forall y \exists x(\varphi(x) \wedge \psi(y))$, but it is not in general equivalent to any prenex formula that would be both $\hat{\Sigma}_{2}^{q}$ and $\hat{\Pi}_{2}^{q}$ (in fact, $\hat{\Sigma}_{2}^{q} \cap \hat{\Pi}_{2}^{q}=\hat{\Sigma}_{1}^{q} \cup \hat{\Pi}_{1}^{q}$ ).

Definition 3.4 For any formula $\varphi$, we define prenex formulas $\varphi^{\exists}$ and $\varphi^{\forall}$ by induction on the complexity of $\varphi$ :

(i) $\varphi^{Q}=\varphi$ if $\varphi$ is open,

(ii) $(C x \varphi)^{Q}=C x \varphi^{C}$,

(iii) $(\neg \varphi)^{Q}=\bigodot \varphi^{\bar{Q}}$,

(iv) $(\varphi \circ \psi)^{Q}=\varphi^{Q} \bigcirc^{Q} \psi^{Q}$,

where $Q, C \in\{\exists, \forall\}$ and $\circ \in\{\wedge, \vee\}$.

Lemma 3.5 Let $\varphi$ be a formula, and $Q \in\{\exists, \forall\}$.

(i) $\left|\varphi^{Q}\right|=|\varphi|$, and $\varphi^{Q}$ is polynomial-time computable given $\varphi$.

(ii) If $\varphi \in \Sigma_{i}^{q}$, then $\varphi^{\exists} \in \hat{\Sigma}_{i}^{q}$. If $\varphi \in \Pi_{i}^{q}$, then $\varphi^{\forall} \in \hat{\Pi}_{i}^{q}$.

(iii) $\varphi^{Q}$ is equivalent to $\varphi$.

Proof: By induction on the complexity of $\varphi$.

Lemma 3.6 Let $\varphi$ be a formula.

(i) If $\varphi \in \Sigma_{i}^{q}$, there are polynomial-time constructible $\hat{G}_{i}^{*}$-proofs of the sequents

$$
\varphi \longrightarrow \varphi^{\exists} ; \quad \varphi^{\exists} \longrightarrow \varphi,
$$

and $\hat{G}_{i}^{\prime *}$-proofs of the sequents

$$
\varphi,(\neg \varphi)^{\forall} \longrightarrow \quad ; \quad \longrightarrow \varphi,(\neg \varphi)^{\forall} .
$$


(ii) If $\varphi \in \Pi_{i}^{q}$, there are polynomial-time constructible $\hat{G}_{i}^{\prime *}$-proofs of the sequents

$$
\varphi \longrightarrow \varphi^{\forall} ; \quad \varphi^{\forall} \longrightarrow \varphi
$$

and $\hat{G}_{i}^{*}$-proofs of the sequents

$$
\varphi,(\neg \varphi)^{\exists} \longrightarrow \quad ; \quad \longrightarrow \varphi,(\neg \varphi)^{\exists} .
$$

Proof: We prove (i) and (ii) by simultaneous induction on the complexity of $\varphi$. The base case of quantifier-free $\varphi$ is straightforward.

The case $\varphi=\exists x \psi$. Note that $\varphi^{\exists}=\varphi^{\forall}=\exists x \psi^{\exists}$ and $(\neg \varphi)^{\exists}=(\neg \varphi)^{\forall}=\forall x(\neg \psi)^{\forall}$. If $\varphi \in \Sigma_{i}^{q}$, we construct the proofs

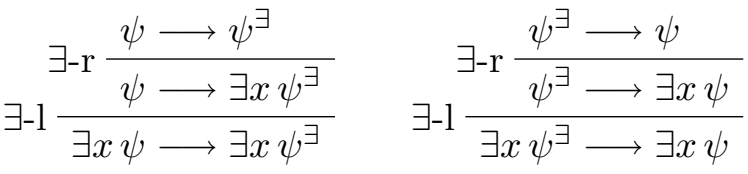

$$
\begin{aligned}
& \exists-1 \frac{\forall-1 \frac{\psi,(\neg \psi)^{\forall} \longrightarrow}{\psi, \forall x(\neg \psi)^{\forall} \longrightarrow}}{\exists x \psi, \forall x(\neg \psi)^{\forall} \longrightarrow} \quad \forall-\mathrm{r} \frac{\longrightarrow \psi,(\neg \psi)^{\forall}}{\longrightarrow \exists x \psi,(\neg \psi)^{\forall}}
\end{aligned}
$$

using the induction hypothesis. If $\varphi \in \Pi_{i}^{q}$, then in fact $\varphi \in \Sigma_{i-1}^{q}$, hence the just constructed proofs in $\hat{G}_{i-1}^{*}$ and $\hat{G}_{i-1}^{\prime *}$ are also valid in both $\hat{G}_{i}^{*}$ and $\hat{G}_{i}^{*}$.

The case $\varphi=\forall x \psi$ is dual.

The case $\varphi=\neg \psi$. Let $\psi \in \Pi_{i}^{q}$, the other case is dual. We construct the proofs

$$
\begin{aligned}
& \neg-\mathrm{r} \frac{\psi,(\neg \psi)^{\exists} \longrightarrow}{(\neg \psi)^{\exists} \longrightarrow \neg \psi} \quad \neg-1 \frac{\longrightarrow \psi,(\neg \psi)^{\exists}}{\neg \psi \longrightarrow(\neg \psi)^{\exists}}
\end{aligned}
$$

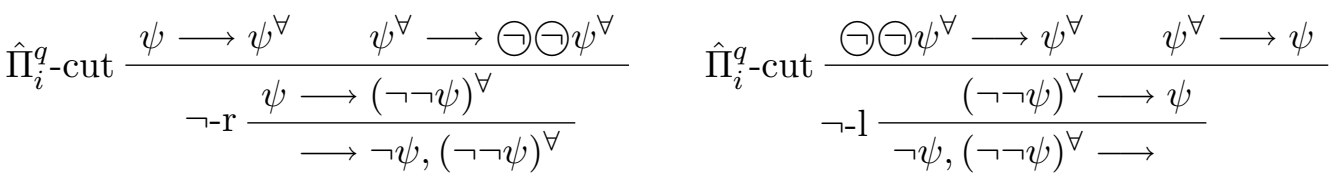

using the induction hypothesis and Lemma 3.3.

The case $\varphi=\psi \wedge \chi$. Let $\psi, \chi \in \Sigma_{i}^{q}$, the other case is similar. Notice that $(\neg(\psi \wedge \chi))^{\forall}=$ $\ominus\left(\psi^{\exists} \oplus^{\exists} \chi^{\exists}\right)$. We construct the proofs

$$
\begin{aligned}
& \hat{\Sigma}_{i}^{q} \text {-cut } \frac{\psi^{\exists}, \chi^{\exists} \longrightarrow \psi^{\exists} \oplus^{\exists} \chi^{\exists} \psi \longrightarrow \psi^{\exists}}{\hat{\Sigma}_{i}^{q} \text {-cut } \frac{\psi, \chi^{\exists} \longrightarrow(\psi \wedge \chi)^{\exists}}{\wedge-1 \frac{\psi, \chi \longrightarrow(\psi \wedge \chi)^{\exists}}{\psi \wedge \chi \longrightarrow(\psi \wedge \chi)^{\exists}}} \chi \chi^{\exists}} \\
& \hat{\Sigma}_{i}^{q} \text {-cut } \frac{\psi^{\exists} \mathbb{\triangle}^{\exists} \chi^{\exists} \longrightarrow \psi^{\exists} \quad \psi^{\exists} \longrightarrow \psi}{\wedge \text {-r } \frac{(\psi \wedge \chi)^{\exists} \longrightarrow \psi}{(\psi \wedge \chi)^{\exists} \longrightarrow \psi \wedge \chi} \quad \hat{\Sigma}_{i}^{q} \text {-cut } \frac{\psi^{\exists} \mathbb{Q}^{\exists} \chi^{\exists} \longrightarrow \chi^{\exists} \quad \chi^{\exists} \longrightarrow \chi}{(\psi \wedge \chi)^{\exists} \longrightarrow \chi}}
\end{aligned}
$$




$$
\begin{aligned}
& \hat{\Pi}_{i}^{q} \text {-cut } \frac{\ominus\left(\psi^{\exists} \oslash^{\exists} \chi^{\exists}\right) \longrightarrow \ominus \psi^{\exists}, \ominus \chi^{\exists} \quad(\neg \psi)^{\forall}, \psi \longrightarrow}{\hat{\Pi}_{i}^{q} \text {-cut } \frac{\psi,(\neg(\psi \wedge \chi))^{\forall} \longrightarrow(\neg \chi)^{\forall}}{\wedge-1 \frac{\psi, \chi,(\neg(\psi \wedge \chi))^{\forall} \longrightarrow}{\psi \wedge \chi,(\neg(\psi \wedge \chi))^{\forall} \longrightarrow}}(\neg \chi)^{\forall}, \chi \longrightarrow}
\end{aligned}
$$

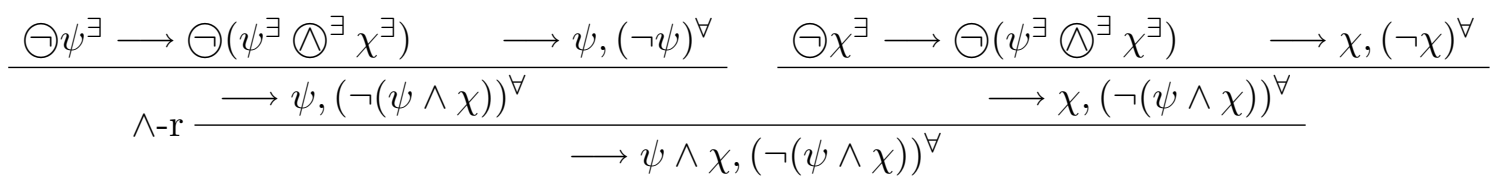

using the induction hypothesis and Lemma 3.3.

The case $\varphi=\psi \vee \chi$ is dual to $\psi \wedge \chi$.

Lemma 3.7 Let $\varphi \in \Sigma_{i}^{q} \cap \Pi_{i}^{q}$.

(i) There are polynomial-time constructible $\hat{G}_{i}^{*}$-proofs of the sequents

$$
\varphi^{\exists},(\neg \varphi)^{\exists} \longrightarrow \quad ; \quad \longrightarrow \varphi^{\exists},(\neg \varphi)^{\exists},
$$

and $\hat{G}_{i}^{\prime *}$-proofs of the sequents

$$
\varphi^{\forall},(\neg \varphi)^{\forall} \longrightarrow \quad ; \quad \longrightarrow \varphi^{\forall},(\neg \varphi)^{\forall} .
$$

(ii) There are polynomial-time constructible $\hat{G}_{i}^{*}$-proofs and $\hat{G}_{i}^{\prime *}$-proofs of the sequents

$$
\varphi^{\exists} \longrightarrow \varphi^{\forall} ; \quad \varphi^{\forall} \longrightarrow \varphi^{\exists} .
$$

Proof: (i): By induction on the complexity of $\varphi$. The case of $\varphi$ open is obvious, and the cases $\varphi=\exists x \psi$ and $\varphi=\forall x \psi$ follow from Lemma 3.3, as $(\neg C x \psi)^{Q}=\bigcirc(C x \psi)^{\bar{Q}}=\bigcirc(C x \psi)^{Q}$.

The case $\varphi=\neg \psi$ : use cut on the induction hypothesis and the sequents $\varphi^{Q} \longrightarrow \curvearrowright \curvearrowright \varphi^{Q}$ and $\left(\curvearrowright \ominus \varphi^{Q} \longrightarrow \varphi^{Q}\right.$ from Lemma 3.3.

The case $\varphi=\psi \wedge \chi$ : note that $(\neg \varphi)^{\exists}=\ominus\left(\psi^{\forall} \oplus^{\forall} \chi^{\forall}\right)$. We prove

$$
\begin{aligned}
& \operatorname{cut} \frac{\longrightarrow \psi^{\exists},(\neg \psi)^{\exists} \quad \operatorname{cut} \frac{\longrightarrow \chi^{\exists},(\neg \chi)^{\exists} \quad \psi^{\exists}, \chi^{\exists} \longrightarrow \psi^{\exists} \bigotimes^{\exists} \chi^{\exists}}{\psi^{\exists} \longrightarrow(\neg \chi)^{\exists},(\psi \wedge \chi)^{\exists}}}{\longrightarrow(\neg \psi)^{\exists},(\neg \chi)^{\exists},(\psi \wedge \chi)^{\exists}}
\end{aligned}
$$

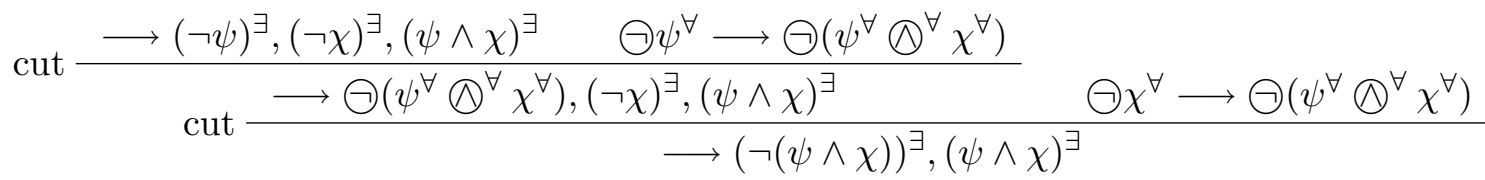

using the induction hypothesis and Lemma 3.3. The other cases are similar.

(ii): We use cut on (i) and Lemma 3.3:

$$
\frac{\varphi^{Q},(\neg \varphi)^{Q} \longrightarrow \curvearrowright \varphi^{\bar{Q}}, \varphi^{\bar{Q}}}{\varphi^{Q} \longrightarrow \varphi^{\bar{Q}}} \quad \frac{\longrightarrow \varphi^{Q},(\neg \varphi)^{Q} \quad \curvearrowright \varphi^{\bar{Q}}, \varphi^{\bar{Q}} \longrightarrow}{\varphi^{\bar{Q}} \longrightarrow \varphi^{Q}}
$$


Lemma 3.8 Let $\alpha$ be an open formula with no occurrence of variables from $X, x \notin X$, and $Q \in\{\exists, \forall\}$.

(i) If $\varphi$ and $\psi$ are prenex formulas, then $\odot \varphi(x / \alpha)=(\odot \varphi)(x / \alpha)$, and $\varphi(x / \alpha) \bigcirc{ }^{Q} \psi(x / \alpha)=$ $\left(\varphi \bigcirc{ }^{Q} \psi\right)(x / \alpha)$, where $\circ \in\{\wedge, \vee\}$.

(ii) $\varphi(x / \alpha)^{Q}=\varphi^{Q}(x / \alpha)$.

Proof: By induction on complexity.

Now we are ready to prove the main theorem.

Proof (of Theorem 2.3): We will show $G_{i} \leq_{p} \hat{G}_{i}$, the other cases are similar. Assume that we are given a $G_{i}$-proof. Define the set $X$ in Definition 3.1 as the set of propositional variables not appearing in the proof. We apply the following transformation to each sequent in the proof:

(i) We negate all $\left(\Pi_{i}^{q} \backslash \Sigma_{i}^{q}\right)$-formulas and move them to the other side of the sequent.

(ii) We replace each $\Sigma_{i}^{q}$-formula $\varphi$ (including those resulting from the previous step) with $\varphi^{\exists}$.

With each transformed sequent we include a short subproof which ensures that the sequent is correctly derived from previous sequents. This subproof will depend on the rule by which the sequent was derived in the original proof.

Initial sequents and structural rules translate to instances of the same rules. Instances of $\left(\Sigma_{i}^{q} \cup \Pi_{i}^{q}\right)$-cut translate to $\hat{\Sigma}_{i}^{q}$-cut.

Assume that a sequent was derived by an instance

$$
\frac{\Gamma, \varphi \longrightarrow \Delta}{\Gamma \longrightarrow \neg \varphi, \Delta}
$$

of $\neg$-r. If $\varphi \notin \Sigma_{i}^{q} \cup \Pi_{i}^{q}$, it translates to a valid instance of $\neg$-r. If $\varphi \in \Pi_{i}^{q} \backslash \Sigma_{i}^{q}$, the assumption and conclusion of the rule translate to the same sequent of the form $\Gamma^{\prime} \longrightarrow(\neg \varphi)^{\exists}, \Delta^{\prime}$. If $\varphi \in \Sigma_{i}^{q}$, we replace the rule with one of the cut inferences

$$
\frac{\Gamma^{\prime}, \varphi^{\exists} \longrightarrow \Delta^{\prime} \longrightarrow \varphi^{\exists},(\neg \varphi)^{\exists}}{\Gamma^{\prime} \longrightarrow(\neg \varphi)^{\exists}, \Delta^{\prime}} \quad \frac{\Gamma^{\prime}, \varphi^{\exists} \longrightarrow \Delta^{\prime} \quad \ominus \ominus \varphi^{\exists} \longrightarrow \varphi^{\exists}}{\Gamma^{\prime},(\neg \neg \varphi)^{\exists} \longrightarrow \Delta^{\prime}}
$$

depending on whether $\varphi$ is in $\Sigma_{i}^{q} \cap \Pi_{i}^{q}$ or $\Sigma_{i}^{q} \backslash \Pi_{i}^{q}$. Here, the sequent $\bigcirc \bigcirc \varphi^{\exists} \longrightarrow \varphi^{\exists}$ comes from Lemma 3.3, and $\longrightarrow \varphi^{\exists},(\neg \varphi)^{\exists}$ is from Lemma 3.7.

The $\neg-\mathrm{l}$ rule is similar.

Consider an instance

$$
\frac{\Gamma \longrightarrow \varphi, \Delta \quad \Gamma \longrightarrow \psi, \Delta}{\Gamma \longrightarrow \varphi \wedge \psi, \Delta}
$$

of $\wedge$-r. If $\varphi, \psi \in \Sigma_{i}^{q}$, we simulate the rule by two cuts with the sequent $\varphi^{\exists}, \psi^{\exists} \longrightarrow \varphi^{\exists} \mathbb{}^{\exists} \psi^{\exists}$ from Lemma 3.3. If $\varphi, \psi \in \Pi_{i}^{q} \backslash \Sigma_{i}^{q}$, we use similarly a cut inference 


$$
\frac{\Gamma^{\prime},(\neg \varphi)^{\exists} \longrightarrow \Delta^{\prime} \quad \frac{\Gamma^{\prime},(\neg \psi)^{\exists} \longrightarrow \Delta^{\prime} \quad \ominus\left(\varphi^{\forall} \bigotimes^{\forall} \psi^{\forall}\right) \longrightarrow \ominus \varphi^{\forall}, \curvearrowright \psi^{\forall}}{\Gamma^{\prime},(\neg(\varphi \wedge \psi))^{\exists} \longrightarrow(\neg \varphi)^{\exists}, \Delta^{\prime}}}{\Gamma^{\prime},(\neg(\varphi \wedge \psi))^{\exists} \longrightarrow \Delta^{\prime}} .
$$

If $\varphi \in \Sigma_{i}^{q} \cap \Pi_{i}^{q}$ and $\psi \in \Pi_{i}^{q} \backslash \Sigma_{i}^{q}$ or vice versa, we use the same inference, but we first derive

$$
\frac{\Gamma^{\prime} \longrightarrow \varphi^{\exists}, \Delta^{\prime} \quad \varphi^{\exists},(\neg \varphi)^{\exists} \longrightarrow}{\Gamma^{\prime},(\neg \varphi)^{\exists} \longrightarrow \Delta^{\prime}}
$$

using Lemma 3.7. In the remaining cases (i.e., either at least one of $\varphi, \psi$ is not in $\Sigma_{i}^{q} \cup \Pi_{i}^{q}$, or one is in $\Sigma_{i}^{q} \backslash \Pi_{i}^{q}$ and the other is in $\left.\Pi_{i}^{q} \backslash \Sigma_{i}^{q}\right)$ the formula $\varphi \wedge \psi$ is not in $\Sigma_{i}^{q} \cup \Pi_{i}^{q}$. We use an instance of the $\wedge$-r rule

$$
\frac{\Gamma^{\prime} \longrightarrow \varphi, \Delta^{\prime} \quad \Gamma^{\prime} \longrightarrow \psi, \Delta^{\prime}}{\Gamma^{\prime} \longrightarrow \varphi \wedge \psi, \Delta^{\prime}}
$$

but if $\varphi$ is in $\Sigma_{i}^{q}$ or $\Pi_{i}^{q} \backslash \Sigma_{i}^{q}$, we first include one of the derivations

$$
\frac{\Gamma^{\prime} \longrightarrow \varphi^{\exists}, \Delta^{\prime} \quad \varphi^{\exists} \longrightarrow \varphi}{\Gamma^{\prime} \longrightarrow \varphi, \Delta^{\prime}} \quad \frac{\Gamma^{\prime},(\neg \varphi)^{\exists} \longrightarrow \Delta^{\prime} \quad \longrightarrow \varphi,(\neg \varphi)^{\exists}}{\Gamma^{\prime} \longrightarrow \varphi, \Delta^{\prime}}
$$

using Lemma 3.6, and similarly for $\psi$.

The other $\wedge$ and $\vee$ rules are handled similarly.

Consider an instance

$$
\frac{\Gamma, \varphi(x / \alpha) \longrightarrow \Delta}{\Gamma, \forall x \varphi \longrightarrow \Delta}
$$

of $\forall$-l, where $\alpha$ is an open formula free for substitution for $x$ in $\varphi$. Note that $\varphi(x / \alpha)^{\forall}=$ $\varphi^{\forall}(x / \alpha)$ by Lemma 3.8. Assume first that $\varphi \in \Pi_{i-1}^{q}$ and $i>1$, so that $\forall x \varphi \in \Sigma_{i}^{q}$. We can derive

$$
\frac{\Gamma^{\prime},(\varphi(\alpha))^{\exists} \longrightarrow \Delta^{\prime} \quad(\varphi(\alpha))^{\forall} \longrightarrow(\varphi(\alpha))^{\exists}}{\forall-1 \frac{\Gamma^{\prime},(\varphi(\alpha))^{\forall} \longrightarrow \Delta^{\prime}}{\Gamma^{\prime},(\forall x \varphi)^{\exists} \longrightarrow \Delta^{\prime}}}
$$

using Lemma 3.7. If $\varphi \in \Pi_{i}^{q} \backslash\left(\Pi_{i-1}^{q} \cup \Sigma_{i}^{q}\right)$, we can derive

$$
\exists-\mathrm{r} \frac{\Gamma^{\prime} \longrightarrow(\neg \varphi(\alpha))^{\exists}, \Delta^{\prime}}{\Gamma^{\prime} \longrightarrow \exists x(\neg \varphi)^{\exists}, \Delta^{\prime}},
$$

noting that $(\neg \forall x \varphi)^{\exists}=\exists x(\neg \varphi)^{\exists}$. If $\varphi \in \Pi_{i}^{q} \cap \Sigma_{i}^{q}$, and $\varphi \notin \Pi_{i-1}^{q}$ or $i=1$, we use the same derivation, but we first infer

$$
\frac{\Gamma^{\prime}, \varphi(\alpha)^{\exists} \longrightarrow \Delta^{\prime} \quad \longrightarrow \varphi(\alpha)^{\exists},(\neg \varphi(\alpha))^{\exists}}{\Gamma^{\prime} \longrightarrow(\neg \varphi(\alpha))^{\exists}, \Delta^{\prime}}
$$

using Lemma 3.7. Otherwise $\varphi \notin \Pi_{i}^{q}$, which means $\forall x \varphi \notin \Sigma_{i}^{q} \cup \Pi_{i}^{q}$. If $\varphi \notin \Sigma_{i}^{q} \cup \Pi_{i}^{q}$, we can directly apply the $\forall$-l rule; if $\varphi \in \Sigma_{i}^{q}$, we first derive 


$$
\frac{\Gamma^{\prime},(\varphi(\alpha))^{\exists} \longrightarrow \Delta^{\prime} \quad \varphi(\alpha) \longrightarrow(\varphi(\alpha))^{\exists}}{\Gamma^{\prime}, \varphi(\alpha) \longrightarrow \Delta^{\prime}}
$$

using Lemma 3.6.

The other quantifier rules are handled in a similar way.

In this way we obtain a $\hat{G}_{i}$-proof of the translation of the end-sequent of the original $G_{i}$-proof. We use cuts with sequents given by Lemma 3.6 to derive the original end-sequent.

\section{References}

[1] Stephen Cook and Tsuyoshi Morioka, Quantified propositional calculus and a second-order theory for $\mathbf{N C}^{1}$, Archive for Mathematical Logic 44 (2005), no. 6, 711-749.

[2] Stephen Cook and Phuong Nguyen, Logical foundations of proof complexity, Cambridge University Press, New York, 2010.

[3] Jan Krajíček, Bounded arithmetic, propositional logic, and complexity theory, Cambridge University Press, 1995.

[4] Jan Krajíček and Pavel Pudlák, Quantified propositional calculi and fragments of bounded arithmetic, Zeitschrift für mathematische Logik und Grundlagen der Mathematik 36 (1990), no. 1, 29-46.

[5] Jan Krajíček and Gaisi Takeuti, On induction-free provability, Annals of Mathematics and Artificial Intelligence 6 (1992), 107-125.

[6] Tsuyoshi Morioka, Logical approaches to the complexity of search problems: Proof complexity, quantified propositional calculus, and bounded arithmetic, Ph.D. thesis, University of Toronto, 2005. 